\title{
Pilot study of the prevalence of binge eating disorder in non-alcoholic fatty liver disease patients
}

\author{
Jinyu Zhanga , Omair Abbasib, Lev Malevanchikc, Neena Mohan ${ }^{a}$, Richard Denicola $^{a}$, \\ Nicholas Tarangeloa, Dina Halegoua-De Marzio ${ }^{d}$
}

Thomas Jefferson University, Philadelphia; University of California at San Francisco, USA

Abstract

Background Non-alcoholic fatty liver disease (NAFLD) is the most common liver disease in the
United States. Binge eating disorder (BED) is the most common form of eating disorder. NAFLD
and BED have similar risk factors, including obesity, insulin resistance, and metabolic syndrome.
The aim of our study was to examine prevalence of BED in NAFLD patients.

Methods We administered the Binge Eating Scale (BES), a questionnaire validated to screen for BED, to NAFLD patients at our Fatty Liver Center. Demographics were retrieved retrospectively from our electronic medical record.

Results Of the total 95 NAFLD patients screened, 22 (23.1\%) had binge eating tendencies; 6 of the $22(6.3 \%)$ scored 27 or more points, suggestive of severe binge eating. Patient demographics included 59 females and 36 males ( 14 females and 8 males positive for BED). Liver disease severity and of metabolic syndrome presence were similar in both groups: 45 patients had steatosis, 25 steatohepatitis, and 24 cirrhosis, of which 10 steatosis, 5 steatohepatitis, and 7 cirrhosis patients screened positive for BED. Of the NAFLD patients with BED, 50.0\% had insulin resistance, $68.2 \%$ hypertension, and $50.0 \%$ hyperlipidemia, whereas among non-BED NAFLD patients $58.9 \%$ had insulin resistance, $63.0 \%$ hypertension, and $67.1 \%$ hyperlipidemia.

Conclusions This pilot study suggests that BED may have a higher prevalence among NAFLD patients than in the general population. Based on these preliminary results, further study into the prevalence of BED is recommended. More data is need to identify effects of BED on the progression of NAFLD and role of BED treatment.

Keywords Non-alcoholic fatty liver disease, binge eating disorder, metabolic syndrome

Ann Gastroenterol 2017; 30 (6): 1-6

\section{Introduction}

Non-alcoholic fatty liver disease (NAFLD) is the most common liver disease in the United States. Obesity is a common and well documented risk factor for NAFLD, and as many as

Departments of a Internal Medicine, Thomas Jefferson University, Philadelphia (Jinyu Zhang, Neena Mohan, Richard Denicola, Nicholas

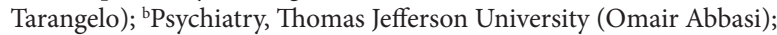
'Internal Medicine, University of California at San Francisco (Lev Malevanchik); ${ }^{\mathrm{d}}$ Gastroenterology and Hepatology, Thomas Jefferson University, Philadelphia (Dina Halegoua-DeMarzio), USA

Conflict of Interest: None

Correspondence to: Dina Halegoua-De Marzio, MD, Department of ${ }^{\mathrm{d} G a s t r o e n t e r o l o g y}$ and Hepatology, Thomas Jefferson University, $132 \mathrm{~S}$. $10^{\text {th }}$ Street, Suite 480, Philadelphia, PA 19107, USA,

e-mail: Dina.Halegoua-DeMarzio@jefferson.edu

Received 6 July 2017; accepted 30 August 2017; published online 10 October 2017

DOI: https://doi.org/10.20524/aog.2017.0200 two thirds of patients with NAFLD are obese [1]. Estimates of the prevalence of NAFLD range from $6.3-33 \%$ worldwide, with a median of $20 \%$ in the general population [2]. The prevalence of non-alcoholic steatohepatitis (NASH) in the US general population has been estimated to be $2-12 \%$ and that of NAFLD to be $20-46 \%[1,3]$. NAFLD is currently the third most frequent indication for liver transplantation, but it has been predicted to become the leading indication by 2020 [4-6].

Binge eating disorder (BED) was made an official diagnosis in 2013 with the release of the Diagnostic and Statistical Manual of Mental Disorders, Fifth Edition (DSM-V) under the category of "Feeding and Eating Disorders." However, it was also present in Appendix B of DSM-IV-TR as a "diagnosis needing more research". BED is the most common eating disorder in the United States, with an estimated lifetime prevalence of $2.6 \%$ among adults-more than the prevalence of bulimia nervosa and anorexia nervosa combined. Patients with BED have recurrent episodes of binge eating, which occur on average at least once a week for three months, and are associated 
Table 1 DSM-V (Diagnostic and Statistical Manual of Mental Disorders, Fifth Edition) criteria for binge eating disorder

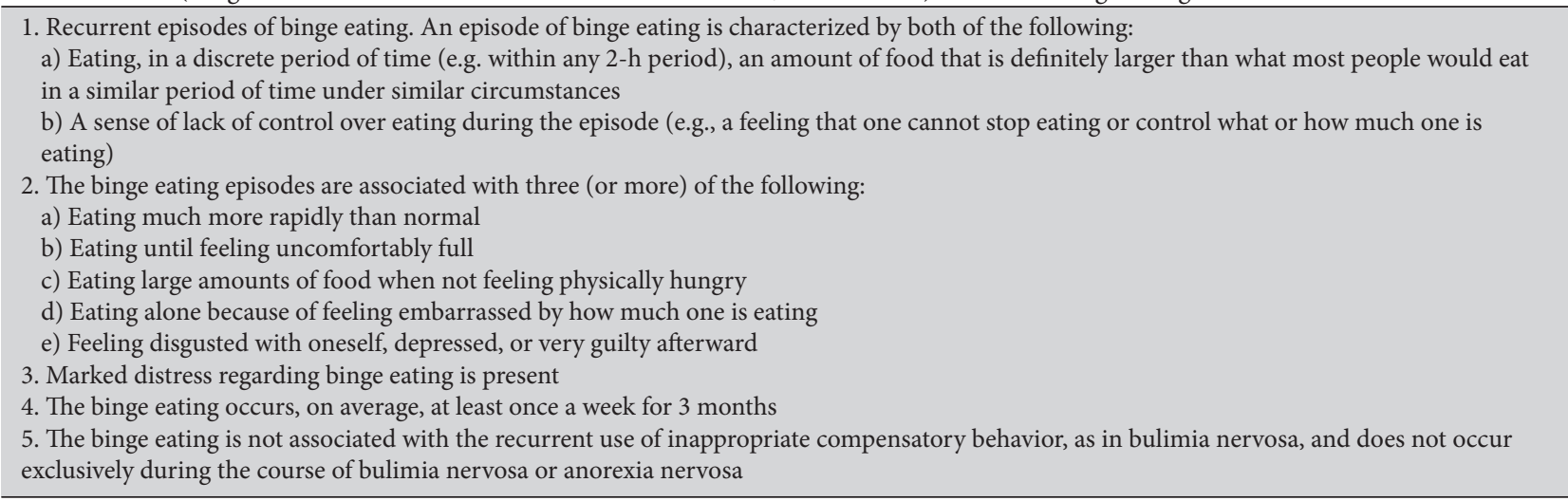

with marked distress (Table 1). Each episode can be defined as eating an amount of food that is definitely larger than what most people would eat in a similar, discrete period of time. A key aspect of binge eating is a sense of lack of control over eating during the episode. Patients may eat alone because of embarrassment at how much they are eating. After an episode, patients may feel very guilty, depressed, or disgusted with themselves. Unlike other eating disorders, individuals with BED do not often partake in inappropriate compensatory behaviors such as inducing vomiting or starvation [7]. Over $40 \%$ of patients with BED are obese [8].

The presence of BED has been associated with a greater risk for developing metabolic syndrome, independent of obesity [9]. BED patients are 13 times more likely to develop type 2 diabetes [10]. In addition, patients with BED have been found to have a lower health-related quality of life and increased healthcare costs when compared to non-BED patients [11]. In view of these issues, the prevalence and effects of BED in vulnerable patient populations have become a focus of research. Studies examining the prevalence of BED amongst patients with type 2 diabetes have found a rate between $1.4 \%$ and $25.6 \%$ [12-16]. Diabetes has also been noted to be an important risk factor in the prognosis of fatty liver disease [17]. Given the shared associations of diabetes and obesity between both BED and NAFLD, the aim of this study was to assess the prevalence and potential effects of BED amongst NAFLD patients.

\section{Patients and methods}

NAFLD patients seeking care at our tertiary Fatty Liver Center between September 2015 and October 2016 were approached following their visit to complete the Binge Eating Scale (BES) in person or were e-mailed a secure online form of the BES. Those that had already completed the BES inclinic were excluded from the online group. In total, we had 36 patients complete the BES in-clinic and 59 complete online, totaling 95 patients.

The BES is a 16-item screening questionnaire that assesses the range of severity of certain behavioral, emotional, and cognitive symptoms associated with BED, with a maximum of 46 points allotted to responses [18]. Since the BES was created prior to the creation of provisional criteria for BED in the DSM, it does not consider the frequency with which binge eating occurs, which is the fourth listed criterion for BED under DSM-V (DMS-V requires that binge eating occur, on average, at least once per week for three months). Therefore, the BES is best used as a screening tool [19].

The BES has been shown to have good internal consistency and validity in studies and across several languages [20-23]. The BES has also been frequently used to assess for BED in patients seeking bariatric surgery [24]. A score of 18 or above is considered positive for binge eating, while a score of 27 or above is indicative of severe binge eating. Using a cutoff of 17, the BES has been shown to have a sensitivity of $0.85-0.98$ and a specificity of 0.48-0.75 [19,25-30].

Descriptive data-race, body mass index (BMI), degree of NAFLD (steatosis, NASH vs. cirrhosis), as well as past medical history for metabolic syndrome (insulin resistance, hyperlipidemia, and hypertension)-were retrieved retrospectively from our electronic medical record. Retrospective determination of degree of NAFLD was based on imaging studies (e.g., ultrasound, magnetic resonance imaging [MRI], computed tomography [CT]), biopsy, or Fibrosure test scores. Patients were determined to have insulin resistance if their hemoglobin a1C was greater than 5.7\% (cutoff for prediabetes) in the past year, they were on diabetes medication, or were documented in the chart as having insulin resistance; hyperlipidemia if they were on statin therapy or documented in the chart as having hyperlipidemia; and hypertension if they were on blood pressure medications or documented in the chart as having hypertension.

\section{Results}

Of the 95 NAFLD patients screened, 22 (23.1\%) had had binge eating tendencies, with 6 of the $22(6.3 \%)$ scoring 27 or more points, suggestive of severe binge eating. Fifty-nine females and 36 males ( 14 females and 8 males screened positive for BED); 82 Caucasians, 8 African Americans, 2 Hispanics, 
and 3 Asians participated (20 Caucasians, 1 African American, and 1 Asian screened positive for BED). The severity of liver disease in both groups-based on biopsies, Fibrosure scores, and liver imaging including CT, ultrasound and MRI-showed 45 patients had hepatic steatosis, $25 \mathrm{NASH}$, and 24 cirrhosis, of which 10 hepatic steatosis, $5 \mathrm{NASH}$ and 7 cirrhosis patients screened positive for BED. A total of 28 patients had biopsies

Table 2 Patient demographics

\begin{tabular}{lcc}
\hline \multirow{2}{*}{ Characteristic } & \multicolumn{2}{c}{$(\mathrm{n}=$ number) $(\%)$} \\
\cline { 2 - 3 } & BED NAFLD & Non-BED NAFLD \\
\hline Caucasian & $20(91.0)$ & $62(85.0)$ \\
African American & $1(4.5)$ & $7(9.6)$ \\
Hispanic & $0(0.0)$ & $2(2.7)$ \\
Asian & $1(4.5)$ & $2(2.7)$ \\
Female & $14(63.6)$ & $45(61.6)$ \\
Male & $8(36.4)$ & $28(38.4)$ \\
Insulin resistance & $11(50.0)$ & $43(58.9)$ \\
Hypertension & $15(68.2)$ & $46(63.0)$ \\
Hyperlipidemia & $11(50.0)$ & $49(67.1)$ \\
\hline
\end{tabular}

* Percentage reflects percentage of sample size with that characteristic

* BED NAFLD is positive binge eating in patients with non-alcoholic fatty liver disease

${ }^{*}$ Non-BED NAFLD is negative binge eating in patients with non-alcoholic fatty liver disease performed. See Table 2 for demographic details. See Fig. 1-3 for severity of liver disease, prevalence of metabolic syndrome, and distribution of BMI.

With respect to the presence of metabolic syndrome in both groups, of the binge eating NAFLD patients $50.0 \%$ had insulin resistance (defined as pre-diabetic and diabetic), $68.2 \%$ had hypertension, and $50.0 \%$ had hyperlipidemia; among the non-binge eating NAFLD patients $58.9 \%$ had insulin resistance, $63.0 \%$ had hypertension, and $67.1 \%$ had hyperlipidemia. The mean BMI for binge eating NAFLD patients was 36.6, median 35.5, range 24.6-53.9, whereas the mean BMI for non-binge eating NAFLD patients was 33.6, median 31.8, range 23.4-62.7.

Statistical analysis included the two-tailed $\mathrm{z}$-score for those that screened positive and those that screened negative in terms of insulin resistance, hypertension and hyperlipidemia. There was no significant difference in terms of insulin resistance $(\mathrm{P}=0.46)$, hypertension $(\mathrm{P}=0.65)$, and hyperlipidemia $(\mathrm{P}=0.1443)$. The two-tailed $t$-score was also determined for BMI. There was no significant difference in BMI between the positive screen and negative screen groups $(\mathrm{P}=0.05)$. With respect to sex, there was no significant difference between males and females: $\mathrm{P}=0.5448$ for the negative screen group and $\mathrm{P}=0.078$ for the positive screen group. The F-test was performed for differences in liver characteristics, i.e., steatosis, $\mathrm{NASH}$, and cirrhosis: $\mathrm{P}=0.086$. No significant variance was found among the different groups between those who screened positive and those who screened negative.

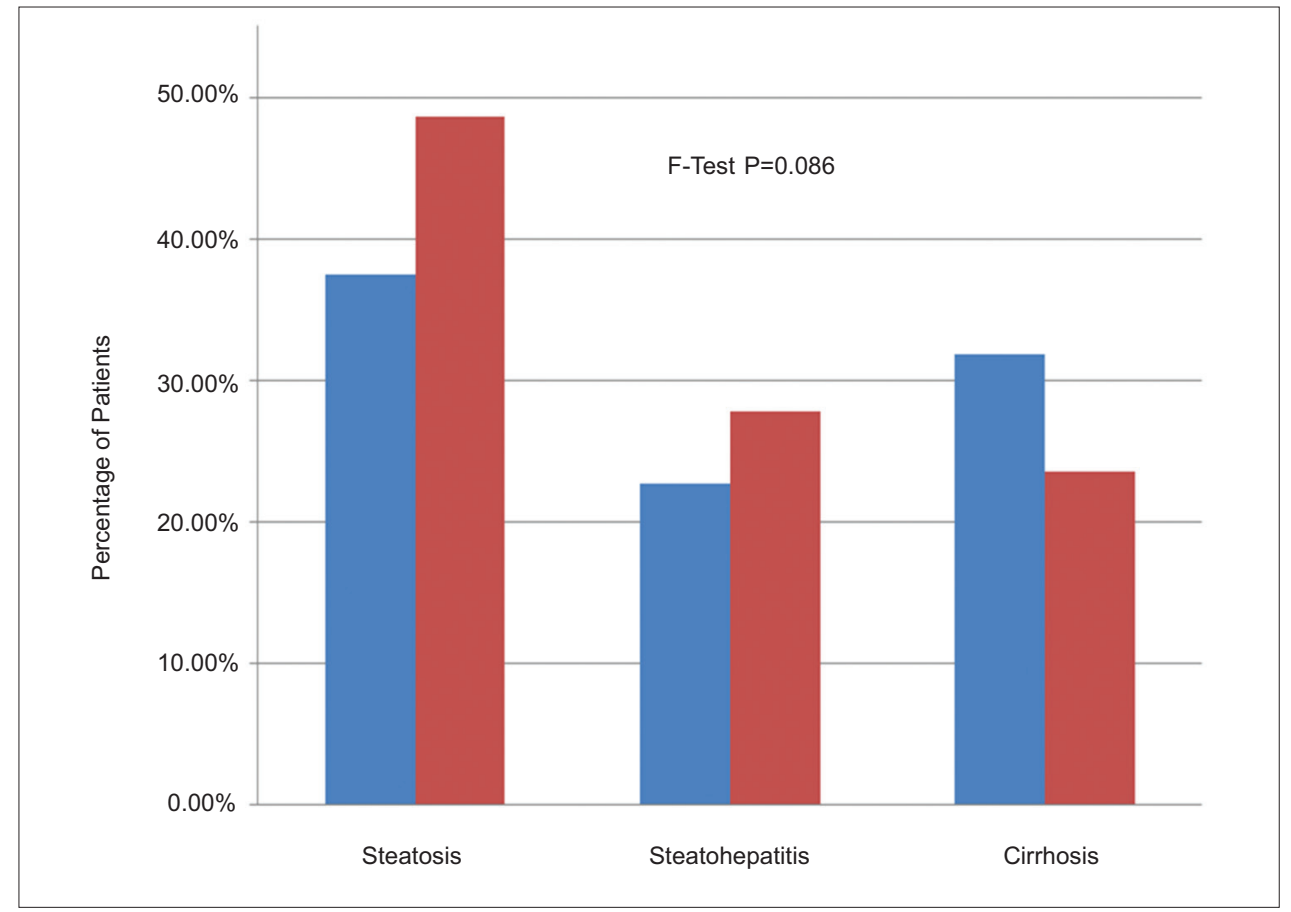

Figure 1 Liver histology in patients with non-alcoholic fatty liver disease

BED NAFLD (positive binge eating in patients with non-alcoholic fatty liver disease)

Non-BED NAFLD (negative binge eating in patients with non-alcoholic fatty liver disease) 


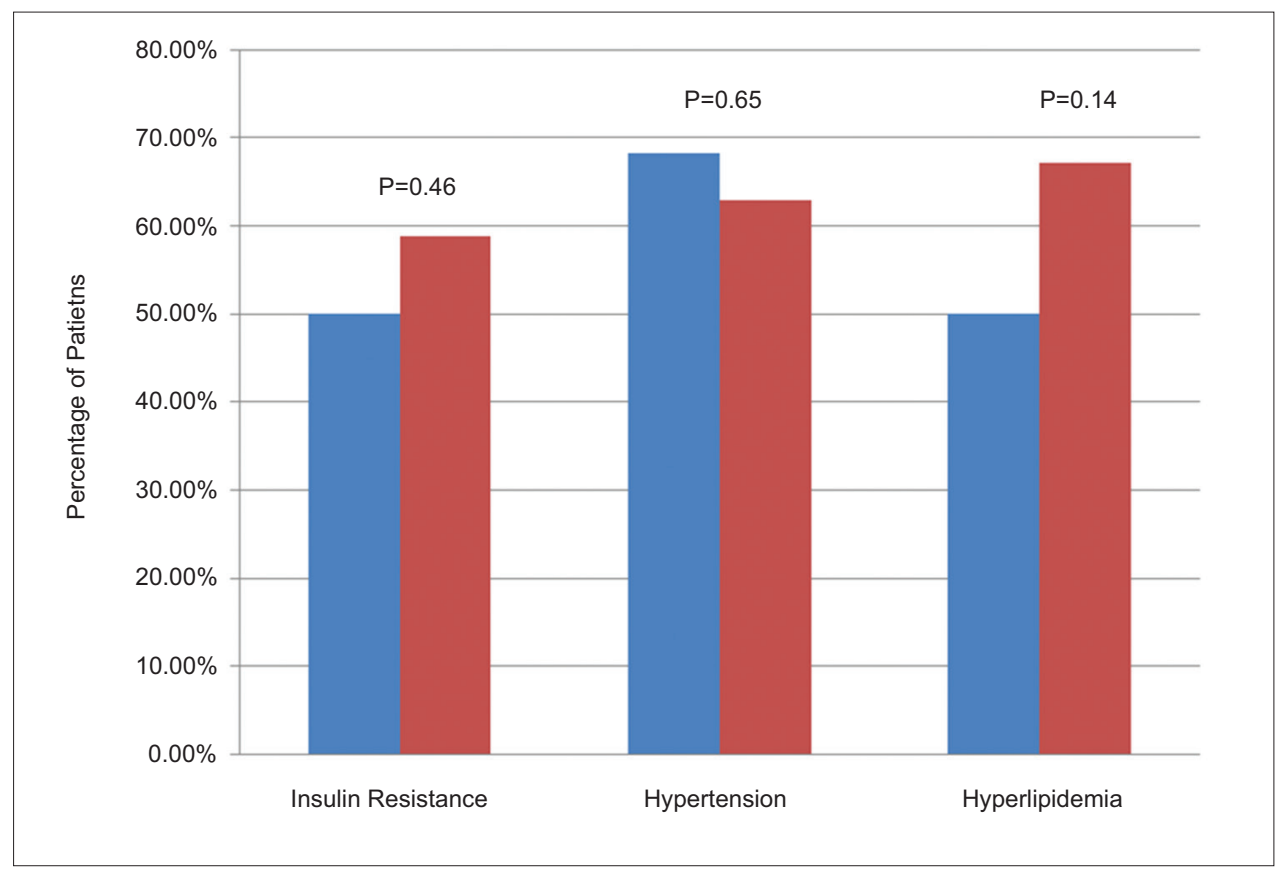

Figure 2 Prevalence of metabolic syndrome in patients with non-alcoholic fatty liver disease BED NAFLD (positive binge eating in patients with non-alcoholic fatty liver disease)

Non-BED NAFLD (negative binge eating in patients with non-alcoholic fatty liver disease)

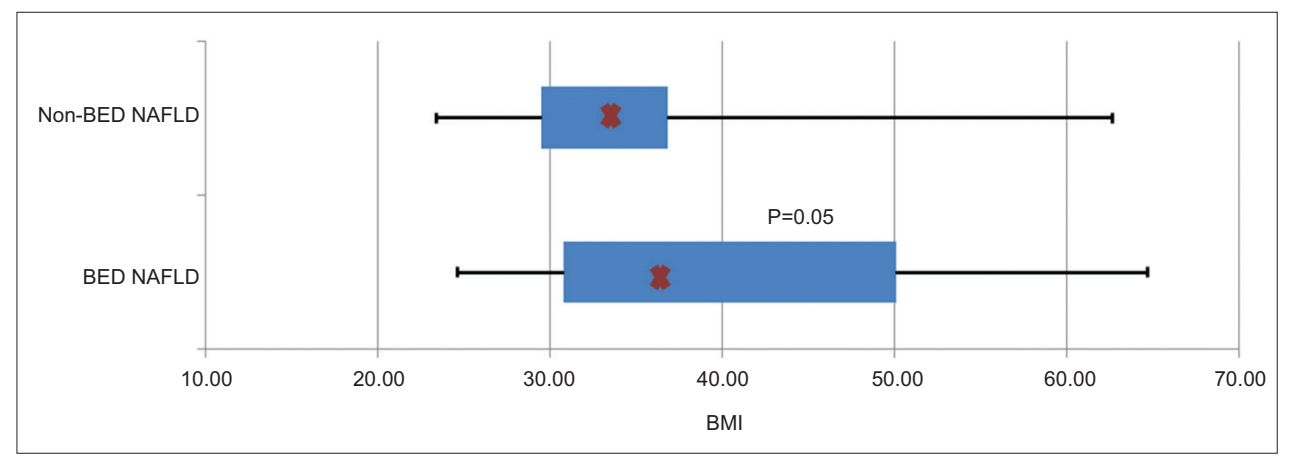

Figure 3 Body mass index (BMI) in patients with non-alcoholic fatty liver disease

Figure 3 is a box and whisker plot of the BMI's in the Non-BED NAFLD (negative binge eating) and BED NAFLD (positive binge eating) patients with non-alcoholic fatty liver disease. The P-value of 0.05 refers to a comparison of the mean BMI values. The " $x$ " represents the mean BMI of each sample population

\section{Discussion}

To our knowledge, this is the first study to evaluate binge eating tendencies in patients with NAFLD. We found that $23.1 \%$ of our subjects screened positive on the BES. While the BES is best utilized as a screening tool and a positive screen is not necessarily equivalent to a diagnosis of $\mathrm{BED}$, looking only at positive screens for BES, a study using the BES in an obese outpatient population found that $14.6 \%$ of men and $10.6 \%$ of women screened positive for BED [25]. Another cross-sectional study utilizing the BES in patients undergoing evaluation for bariatric surgery found that $17 \%$ of patients screened positive [20]. In both studies, the percentage of people who screened positive was well below our $23.1 \%$. Two studies correlating the BES with a confirmed diagnosis of BED found that, if the cutoff score is raised to 27 , the specificity of the BES increased to about $95 \%[25,26]$. Utilizing the higher cutoff score, our study would screen $6.3 \%$ of NAFLD patients positive for BED, which is still above what is seen in the general population $(2.6 \%)$ [8].

Analysis of our data is limited by the fact that BED is new to DSM-V, although it was characterized in DSM-IV and estimates in the existing literature are based on DSM-IV criteria. BMIs for those who completed the BES in-clinic $(n=36)$ were taken at the time of BES completion, whereas for those who completed the BES online $(n=59)$ the BMI was obtained as close as possible 
to the date of BES completion. Therefore, there may exist some discrepancy in BMI reporting between the two groups. While there were no statistically significant differences seen with regard to metabolic syndrome, analysis is limited by the retrospective nature of our data. However, all patients included in the study were seen in the liver clinic within the year prior to BES completion. While there was no significant variance in histology (steatosis, NASH, vs. cirrhosis), the analysis was limited by the retrospective nature of our data: we could only evaluate the examinations that had been previously performed, whether imaging or some other modality. Only close to one third of our patients had biopsies.

In general, this study was intended to provide pilot data concerning the possibility of a greater prevalence of binge eating symptoms, if not BED, in the NAFLD population. Current criteria in the DMS-V for BED measure severity by the number of such episodes per week. The BES attempts to assess severity of BED by the presence of certain psychological, emotional, and behavioral symptoms; it does so by measuring how intense the symptoms feel to the patient and not by the frequency of events.

Currently, no data exist on the connection between BED and NAFLD. Our study suggests that there could be a possible connection, given the percentage of positive screens in our sample population. Further case-controlled studies with a larger sample size are needed. This could be an important area of interest to explore further, as it would point to a need for additional assessment of eating patterns in the NAFLD population. Often patients turn to popular diet programs for weight loss, many of which rely on restrictive eating patterns. Dietary restraint has been shown to be a behavior that may perpetuate binge eating behavior, rather than preventing

\section{Summary Box}

\section{What is already known:}

- Nonalcoholic fatty liver disease (NAFLD) is the most common liver disease in the United States (US)

- Binge eating disorder (BED) carries an estimated prevalence of $2.6 \%$ among US adults; it is thereby the most common form of eating disorder

- BED patients are 13 times more likely to develop type 2 diabetes

\section{What the new findings are:}

- BED may have a higher prevalence in patients with NAFLD compared to the general population

- More data will be needed to identify the effects of BED on the progression of NAFLD and the role of BED treatment it [31]. Identifying such individuals and providing closer nutritional guidance along with addressing eating dysregulated eating behaviors may lead to better weight loss. Currently, psychotherapeutic interventions have shown efficacy for BED, including cognitive behavioral therapy and mindfulness, which is not standard in NAFLD treatment. In addition there is promising evidence supporting certain anticonvulsant therapies, stimulants, and appetite suppressants for the management of BED [32,33]. Our findings leave open the possibility that treatment of BED in a patient with BED and NAFLD could lead to an improvement in the patient's liver disease. A larger multicenter cross-sectional study is needed to identify prevalence rates of BED in NAFLD. In addition, a case-controlled prospective study of NAFLD progression patients with and without comorbid BED would provide additional data concerning the effects of BED on fatty liver disease outcomes.

\section{References}

1. Williams CD, Stengel J, Asike MI, et al. Prevalence of nonalcoholic fatty liver disease and nonalcoholic steatohepatitis among a largely middle-aged population utilizing ultrasound and liver biopsy: a prospective study. Gastroenterology 2011;140:124-131.

2. Chalasani N, Younossi Z, Lavine JE, et al. The diagnosis and management of non-alcoholic fatty liver disease: practice Guideline by the American Association for the Study of Liver Diseases, American College of Gastroenterology, and the American Gastroenterological Association. Hepatology 2012;55:2005-2023.

3. Sayiner M, Koenig A, Henry L, Younossi ZM. Epidemiology of nonalcoholic fatty liver disease and nonalcoholic steatohepatitis in the United States and the rest of the world. -Clin Liver Dis 2016;20:205-214.

4. Ratziu V, Bellentani S, Cortez-Pinto H, Day C, Marchesini G. A position statement on NAFLD/NASH based on the EASL 2009 special conference. J Hepatol 2010;53:372-384.

5. Sanyal AJ, Brunt EM, Kleiner DE, et al. Endpoints and clinical trial design for nonalcoholic steatohepatitis. Hepatology 2011;54:344-353.

6. Wree A, Broderick L, Canbay A, Hoffman HM, Feldstein AE. From NAFLD to NASH to cirrhosis-new insights into disease mechanisms. Nat Rev Gastroenterol Hepatol 2013;10:627-636.

7. Citrome L. A primer on binge eating disorder diagnosis and management. CNS Spectr 2015;20 Suppl 1:44-50.

8. Kessler RC, Berglund PA, Chiu WT, et al. The prevalence and correlates of binge eating disorder in the World Health Organization World Mental Health Surveys. Biol Psychiatry 2013;73:904-914.

9. Hudson JI, Lalonde JK, Coit CE, et al. Longitudinal study of the diagnosis of components of the metabolic syndrome in individuals with binge-eating disorder. Am J Clin Nutr 2010;91:1568-1573.

10. Raevuori A, Suokas J, Haukka J, et al. Highly increased risk of type 2 diabetes in patients with binge eating disorder and bulimia nervosa. Int J Eat Disord 2015;48:555-562.

11. Ágh T, Kovács G, Pawaskar M, Supina D, Inotai A, Vokó Z. Epidemiology, health-related quality of life and economic burden of binge eating disorder: a systematic literature review. Eat Weight Disord 2015;20:1-12.

12. Wing RR, Marcus MD, Epstein LH, et al. Binge eating in obese patients with type II diabetes. Int J Eat Disord 1989;8:671-679.

13. Crow S, Kendall D, Praus B, Thuras P. Binge eating and other psychopathology in patients with type II diabetes mellitus. Int J Eat 
Disord 2001;30:222-226.

14. Allison KC, Crow SJ, Reeves RR, et al. Binge eating disorder and night eating syndrome in adults with type 2 diabetes. Obesity (Silver Spring) 2007;15:1287-1293.

15. Herpertz S, Albus C, Lichtblau K, Köhle K, Mann K, Senf W. Relationship of weight and eating disorders in type 2 diabetic patients: a multicenter study. Int J Eat Disord 2000;28:68-77.

16. Mannucci E, Tesi F, Ricca V, et al. Eating behavior in obese patients with and without type 2 diabetes mellitus. Int J Obes Relat Metab Disord 2002;26:848-853.

17. Wanless IR, Lentz JS. Fatty liver hepatitis (steatohepatitis) and obesity: an autopsy study with analysis of risk factors. Hepatology 1990; 12:1106-1110.

18. Gormally J, Black S, Daston S, Rardin D. The assessment of binge eating severity among obese persons. Addict Behav 1982;7:47-55.

19. Celio AA, Wilfley DE, Crow SJ, Mitchell J, Walsh BT. A comparison of the binge eating scale, questionnaire for eating and weight patterns-revised, and eating disorder examination questionnaire with instructions with the eating disorder examination in the assessment of binge eating disorder and its symptoms. Int $J$ Eat Disord 2004;36:434-444.

20. Brunault P, Gaillard P, Ballon N, et al. [Validation of the French version of the Binge Eating Scale: Examination of its factor structure, internal consistency and construct validity in a non-clinical and a clinical population]. Encephale 2016;42:426-433.

21. Robert, S. A., Rohana, A. G., Suehazlyn, Z., Maniam, T., Azhar, S. S., \& Azmi, K. N. (2013). The validation of the malay version of binge eating scale: a comparison with the structured clinical interview for the DSM-IV. Journal of Eating Disorders, 1, 28.

22. Imperatori C, Innamorati M, Lamis DA, et al. Factor structure of the binge eating scale in a large sample of obese and overweight patients attending low energy diet therapy. Eur Eat Disord Rev
2016;24:174-178.

23. Timmerman GM. Binge Eating Scale: further assessment of validity and reliability. J Appl Biobehav Res 1999;4:1-12.

24. Niego SH, Kofman MD, Weiss JJ, et al. Binge eating in the bariatric surgery population: A review of the literature. Int J Eat Disord 2007;40:349-359.

25. Ricca V, Mannucci E, Moretti S, et al. Screening for binge eating disorder in obese outpatients. Compr Psychiatry 2000;41:111-115.

26. Grupski AE, Hood MM, Hall BJ, Azarbad L, Fitzpatrick SL, Corsica JA. Examining the Binge Eating Scale in screening for binge eating disorder in bariatric surgery candidates. Obes Surg 2013;23:1-6.

27. Freitas SR, Lopes CS, Appolinario JC, Coutinho W. The assessment of binge eating disorder in obese women: a comparison of the binge eating scale with the structured clinical interview for the DSM-IV. Eat Behav 2006;7:282-289.

28. Greeno CG, Marcus MD, Wing RR. Diagnosis of binge eating disorder: Discrepancies between a questionnaire and clinical interview. Int J Eat Disord 1995;17:153-60.

29. Meneghini LF, Spadola J, Florez H. Prevalence and associations of binge eating disorder in a multiethnic population with type 2 diabetes. Diabetes Care 2006;29:2760.

30. Blomquist KK, Milsom VA, Barnes RD, et al. Metabolic syndrome in obese men and women with binge eating disorder: developmental trajectories of eating and weight-related behaviors. Compr Psychiatry 2012;53:1021-1027.

31. Marcus MD, Wing RR, Lamparski DM. Binge eating and dietary restraint in obese patients. Addict Behav 1985;10:163-168.

32. Peat CM, Brownley KA, Berkman ND, Bulik CM. Binge eating disorder: Evidence-based treatments. Curr Psychiatr 2012;1 1:32-39.

33. Brownley K, Berkman N, Peat C, et al. Binge-eating disorder in adults: a systematic review and meta-analysis. Ann Intern Med 2016;165:409-420. 